\title{
Image rotation of misoriented letter strings: Effects of orientation cuing and repetition
}

\author{
KEVIN JORDAN \\ San Jose State University, San Jose, California \\ and \\ LAREE A. HUNTSMAN \\ University of Wisconsin, Milwaukee, Wisconsin
}

\begin{abstract}
Three experiments were designed to investigate whether the characteristic function relating response time to stimulus orientation reflects the observer imagining the rotation of the stimulus to upright (the "image rotation" hypothesis) or rotation of an internal reference frame in response to the misoriented stimulus (the "frame rotation" hypothesis). Identification times in response to misoriented words were measured in Experiment 1, whereas in Experiments 2 and 3 , lexical decision times in response to misoriented letter strings were measured. Trials occurred in blocks; words within a block were presented at the same orientation. It was argued that this mode of presentation would facilitate the use of a frame rotation strategy by allowing for a gradual readjustment of an internal reference frame. The characteristic "mental rotation" function was observed in all three experiments. However, the data indicated that observers continued to imagine the rotation of the word to upright in each trial; there was no evidence of readjustment of an internal reference frame. An additional finding of interest occurred in Experiment 1, in which observers identified the same set of misoriented words across two sessions. The identifica. tion times were faster, and the slope of the mental rotation function was lower, in the second session. These results are discussed as in relation to the image rotation hypothesis of mental rotation and to "instance-based skill acquisition" (Masson, 1986) in word recognition.
\end{abstract}

In the present paper, we examine an issue raised by Koriat and Norman (1984) in the title of their paper "What is rotated in mental rotation?" As an example of research that is usually interpreted as indicating "mental rotation," Cooper and Shepard (1973) reported that the time to decide whether an alphanumeric character was in its normal or mirror-image version increased monotonically as the angular deviation from upright increased to $180^{\circ}$. Furthermore, this monotonic increase persisted when observers were cued to stimulus orientation prior to each trial. Cooper and Shepard proposed that subjects mentally rotated some internal representation of the alphanumeric to its normal, upright position prior to making a decision about the version of the stimulus. This "normalization" process has been interpreted as supportive of a general class of template models of form perception which

Preparation of this report was supported by NASA Cooperative Agreement NCC 2-327 to San Jose State University, Kevin Jordan, Project Director. We would like to thank Pierre Jolicoeur, Asher Koriat, Lester Krueger, and Roger Shepard for many helpful comments on earlier versions of this manuscript. In particular, Pierre Jolicoeur and Asher Koriat suggested the random presentation conditions used in Experiments 2 and 3. Additional thanks go to Susanne Delzell, Kimberly Jobe, Fidel Lam, and Beverly Sanford for their assistance with data collection, John Empey for his technical assistance, and especially Guy Woffindin for programming Experiments 2 and 3. Requests for reprints should be sent to Kevin Jordan, Department of Psychology, San Jose State University, San Jose, CA 95192. propose that our stored representations of form in memory are orientation-specific (see Jolicoeur, 1985).

\section{Image versus Frame Rotation}

The interpretation that subjects normalize an internal image prior to deciding on its version is not the only plausible interpretation of the function that relates reaction time to orientation. For example, it is also possible that observers rotate an internal frame of reference into congruence with the misoriented stimulus item (Koriat \& Norman, 1984; Robertson, Palmer, \& Gomez, 1987). Specifically, an observer who is presented with a misoriented stimulus could imagine the rotation of that stimulus to upright (the image rotation hypothesis) or rotate an internal frame of reference into congruence with the misoriented stimulus (the frame rotation hypothesis) for further processing.

In order to test between these hypotheses, Koriat and Norman (1984; Experiment 2) analyzed lexical decision response times to misoriented Hebrew letter strings as a function of their angular departure from upright (ADU) and angular departure from the preceding stimulus orientation (ADP). The logic of the test was that if observers rotate the image to its normal orientation prior to making a lexical decision, reaction times would be a function of ADU. Conversely, if observers rotate an internal reference frame into congruence with the stimulus, then reaction times would be a function of the ADP, since the frame 
would only have to be moved from the previous trial's orientation to the present trial's orientation. The data indicated that reaction times were largely dependent on ADU, thus supporting the image rotation hypothesis, although there was a small, but significant, effect of ADP. However, a crucial assumption of the test was that observers "leave" their reference frame at the previous orientation, rather than return it to the possible default upright orientation. To control for this possibility, Koriat and Norman (1984, Experiment 3A) decreased their intertrial interval to $20 \mathrm{msec}$ to prevent the return of the frame to upright, and they repeated the experiment. The lexical decision times were again largely dependent on $\mathrm{ADU}$, further supporting the image rotation hypothesis.

Given a small but significant effect of ADP in their Experiment 2, Koriat and Norman (1984) concluded that both image and frame rotation strategies are available to the observer, but that observers use the image rotation strategy because it is more expedient. They therefore attempted to make the frame rotation strategy more expedient by manipulating the sequential dependencies among the trials (Koriat \& Norman, 1984, Experiment 4). Observers were again asked to make lexical decisions about Hebrew letter strings, but the orientation of the letter string on a given trial was $80 \%$ predictive of the orientation of the letter string on the following trial. It was expected that observers would use the frame rotation strategy due to its expedience. However, the lexical decision times again were largely dependent on ADU's favoring the image rotation hypothesis.

Robertson et al. (1987) also attempted to test between the image and frame rotation hypotheses by examining reaction times required to make a decision about the version (normal or reflected) or identity of letters as a function of both absolute and relative orientation. The major procedural refinement was that Robertson et al. included two stimuli in a given trial. Once the response to the first stimulus was made, a second stimulus was presented $100 \mathrm{msec}$ later. Thus, Robertson et al. used a shortened response-stimulus interval to determine whether the subjective reference frame was at the orientation of the first stimulus (frame rotation hypothesis) or at upright (image rotation hypothesis). Like Koriat and Norman (1984), Robertson et al. found effects of both absolute and relative orientation. They interpreted the data as supportive of a probability mixture model proposing that both image and frame rotation strategies are available to an observer. The strategy used depends on several factors, such as the orientation disparity between the two stimuli within a given trial and whether the two stimuli were both reflected or both normal.

Except for the one experiment by Koriat and Norman (1984) in which the sequential dependencies were manipulated (Experiment 4), a common feature of all of the experiments reported above is the randomized presentation of stimulus orientations. This randomized presentation induces uncertainty from trial to trial about the upcoming stimulus orientation. As a result, randomized presenta- tion would prevent the gradual adaptation of a reference frame to a particular stimulus orientation. If both image and frame rotation strategies are available to the observer, the randomized presentation of stimulus orientations would bias the data toward an image rotation strategy for at least two reasons. First, the uncertainty concerning stimulus orientation on upcoming trials would encourage observers to default to a strategy of image rotation, since this would be the most expedient strategy; that is, if orientations from $0^{\circ}$ to $360^{\circ}$ are randomly sampled across trials, to leave an internal reference frame at either $0^{\circ}$ or $180^{\circ}$ would minimize the total amount of angular mental rotation across all trials. Given a lifetime of $0^{\circ}$ reading, it would make sense for the internal reference frame to default to $0^{\circ}$ under conditions of randomized presentation of orientations. Second, it might well be that frame rotation occurs, but that it takes repeated exposure (adaptation) for a readjustment of this internal reference frame; that is, it might be expected that more than one trial is required for an internal reference frame to be readjusted. Thus, randomized presentation of orientations would prevent readjustment of an internal reference frame, under the assumption that this readjustment requires repeated exposure to the same orientation.

We proposed to remove the above biases by presenting observers with blocks of trials in a mental rotation task in which all stimuli within a given block had the same orientation. Thus, within a block, there would be no trialto-trial uncertainty regarding stimulus orientation, and this constancy of orientation would allow readjustment of an internal reference frame. If a frame rotation strategy was available to an observer, blocked presentation of orientations should have encouraged its use.

\section{The Present Studies}

In order to test between the image and frame rotation hypotheses, it is necessary to have a combination of a stimulus set and a task in which reaction times increase monotonically with stimulus misorientation. Corballis and Cullen (1986) suggest that a "criterion for deciding whether or not mental rotation was the dominant strategy under any given condition was the shape of the function relating [response] time to angular orientation"' (p. 29). For example, on the basis of their monotonically increasing function relating reaction times to stimulus orientation, Cooper and Shepard (1973) estimated that the rotation rate for alphanumeric characters was approximately $360^{\circ}$ per second. As an additional criterion, Cooper and Podgorny (1976, p. 510) state that the rate of mental rotation does not increase with practice. However, there are cases in the literature in which reaction times decrease (which could indicate a change in the rate of mental rotation) with stimulus repetition (e.g., Jolicoeur, 1985; Jolicoeur \& Milliken, 1989; Masson, 1986). These specific transfer effects, which could potentially affect the interpretation of any blocking effect, can be avoided by choosing a stimulus set large enough to prevent the need for stimulus repetition within an experimental session. 
One stimulus set and task combination that meets these criteria is the identification of misoriented words. Jordan (1987) reported that the time to name high-frequency English words increased monotonically as the orientation of the words changed from $0^{\circ}$ to $180^{\circ}$ in $30^{\circ}$ steps. The overall estimated rotation rate was $403^{\circ}$ per second, which is close to the rate reported by Cooper and Shepard (1973). In addition, without stimulus repetition, the "mental rotation" function persisted across several replications of the experimental design; there was no practice effect.

\section{EXPERIMENT 1}

Based on the above, Experiment 1 was designed to test whether or not mental rotation of words occurs, and, if so, whether the image or an internal reference frame was rotated. As noted above, earlier attempts to distinguish between the image and frame rotation strategies were focused on shortening the intertrial interval and examining reaction times to a particular stimulus as a function of the preceding stimulus orientation (Koriat \& Norman, 1984; Robertson et al., 1987). Given that these studies failed to yield compelling evidence for a frame rotation effect

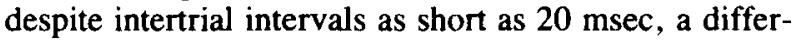
ent approach seemed necessary if a frame effect was to be observed. Our approach was to remove the uncertainty about stimulus orientation across trials that had been produced by the randomized presentation of orientations in previous studies. This was accomplished by presenting observers with blocks of trials in which the stimulus orientation on the first trial of a given block was the same as the stimulus orientation for all other trials within the block. If the frame rotation strategy is available to an observer, this blocking manipulation should encourage its use in that there will be no trial-to-trial uncertainty concerning word orientation, which will allow for a readjustment of an internal frame of reference within a given block of trials.

An additional variable included in Experiment 1 was the shape of the frame within which a word was presented in the tachistoscope. With this manipulation, we sought to determine whether or not the "mental rotation" of words could be affected by providing the observer with spatial axes defining upright (i.e., a rectangular viewing frame). As Corballis, Nagourney, Shetzer, and Stefanatos (1978) have pointed out, rectangular frames might facilitate mental rotation by giving cues to the local horizontal and vertical axes, whereas with circular frames, observers may be "more uncertain about where to rotate the characters.... This may have slowed their mental rotation rate" (p. 265). They reported that the rate of mental rotation of alphanumerics presented within a circular frame appeared to be slower than the rate of mental rotation reported by Corballis, Zbrodoff, and Roldan (1976) for the same stimuli presented within a rectangular frame. Given that this comparison was made across studies, there was no statistical evaluation of the relative rates of rotation for the two frame types. Therefore, in Experiment 1, observers participated in two sessions; in the first session, they viewed the stimulus words within a circular (or rectangular) frame, and in the second session, they viewed the words within a rectangular (or circular) frame.

\section{Method}

Observers. Twelve students from an introductory psychology course at San Jose State University participated in the experiment in order to earn course credit.

Stimuli and Apparatus. The observers were asked to identify words presented one at a time in a four-channel projection tachistoscope. A pool of 140 words was drawn from a list of high-frequency words (appearing more than 50 times per million words of text; Zechmeister \& Nyberg, 1982, Appendix, Table G) and ranged from four to seven letters in length. Words were randomly assigned to orientations to balance possible effects of word length on identification time. The viewing distance was $78.7 \mathrm{~cm}$ and the letters were $1 \mathrm{~cm}\left(0.7^{\circ}\right) \mathrm{high}$. For one of the two sessions, the black uppercase letters were centered on a rectangular, white background $8.8 \mathrm{~cm}$ $\left(6.4^{\circ}\right)$ high and $12.6 \mathrm{~cm}\left(9.1^{\circ}\right)$ wide. This viewing frame remained environmentally and egocentrically upright throughout the session. For the other session, the words were centered on a white, circular background $8 \mathrm{~cm}\left(6.4^{\circ}\right)$ in diameter. The words were presented at one of six orientations $-0^{\circ}$ to $150^{\circ}$, in $30^{\circ}$ steps-relative to their normal, upright orientation.

Procedure. The observers were run individually in a small experimental room. They were told to look through the viewing hood of the apparatus and to identify a word that was to be presented as rapidly as possible while maintaining accuracy. They first proceeded through 18 practice trials. A trial consisted of a 500 msec presentation of a fixation cross (rectangular frame condition) or fixation circle (circular frame condition), followed immediately by the presentation of the word to be identified for up to $3 \mathrm{sec}$. The timer started when the word was presented. When the observer identified the word, the voice activated a relay that stopped the timer and terminated the stimulus. The intertrial interval was approximately $3 \mathrm{sec}$.

Following the practice trials, the observers proceeded through six blocks of 18 trials-one block for each of the six orientations. The observers were told that for all 18 trials within a block, the words would be at the same orientation. The observers were therefore cued to the orientation of the remaining 17 trials in a block by the first trial. The order of presentation of the six blocks of orientations was counterbalanced across observers, using a Latin square. A response was considered an error if the observer misidentified the word or made a sound that stopped the timer prior to identifying the word. Error trials were replaced, using new words at the end of the block in which they occurred.

Each observer participated in two sessions of 18 practice and 108 test trials (six blocks of 18 ) separated by approximately $48 \mathrm{~h}$. Within a given session, all words were presented within the same frame type. Six of the 12 observers were presented with words in a rectangular frame in the first session and with words in a circular frame in the second session. This order was reversed for the remaining 6 observers. It is important to note that observers never saw the same word twice within a session, but that the same pool of words at the same orientations was used in both sessions. Each session lasted approximately $35 \mathrm{~min}$.

\section{Results and Discussion}

The identification times were analyzed in a 2 (counterbalanced orders of viewing frames across days) $\times 2$ (viewing frames) $\times 6$ (orientations) $\times 18$ (replications) $\times 12$ (observers) mixed analysis of variance. Order was a between-subjects factor. 
Figure 1 presents the mean identification times as a function of word orientation and viewing frame. The main effect of orientation was statistically significant $[F(5,50)$ $=18.66, p<.01]$. Clearly, the functions for both frame conditions satisfy the Corballis and Cullen (1986) criterion for a mental rotation function described above; identification times increased with increasing angular departure from the normal, upright orientation.

The number of errors occurring throughout the 216 test trials ranged from 3 to 16 across the 12 observers, with an average of 6 errors per observer (overall error rate of $2.8 \%$ ). The highest error rate was for words oriented at $120^{\circ}(4.9 \%)$, followed by words at $150^{\circ}(4.4 \%)$. Thus, there was no evidence for a speed-accuracy tradeoff.

A frame rotation strategy would have been indicated by an orientation $\times$ replications interaction. Specifically, the slope of the orientation function would have decreased across replications. This interaction was significant $[F(85,850)=1.55, p<.01]$, but the pattern of means was the opposite of that expected if observers were able to gradually readjust an internal reference frame within a block of trials. The identification times increased within a block of 18 trials [the effect of replications was significant, $F(17,170)=3.09, p<.01$ ], and they increased more with increasing angular departure of the words from upright (the orientation $\times$ replications interaction). Thus, it seems that despite the cuing of upcoming orientations across several trials, observers imagined the rotation of a misoriented word to upright on each trial. The replications effect suggests that this image rotation process may be subject to fatigue. Alternatively, observers might have had difficulty maintaining vigilance, given the relatively long (3-sec) intertrial interval.

In addition, because the first trial of a given block cued the observer to the orientation of the remaining 17 trials, the immediate readjustment of an internal reference frame would have been indicated by a decrease in identification times from Trial 1 to Trial 2 within a block. However, the mean for the first trial within a block $(M=719 \mathrm{msec})$ was statistically indistinguishable from the mean for the second trial $(M=729 \mathrm{msec})$.

The three-way interaction of order of frames across days, viewing frame, and orientation was statistically significant $[F(5,50)=9.58, p<.01]$. The effects of frame and orientation were dependent on the order of presentation of the viewing frames across test days; identification times were faster on the 2 nd day regardless of viewing frame. Because there was no effect of viewing frame (see below), and in order to examine this interaction more closely, the identification times were collapsed across levels of viewing frame and analyzed in a 2 (days) $\times 6$ (orientations) $\times 18$ (replications) $\times 12$ (observers) withinsubjects analysis of variance. In this analysis, the effect of orientation was again statistically significant $[F(5,55)$ $=19.62, p<.01]$, as was the main effect of days $[F(1,11)=11.81, p<.01]$. The orientation $\times$ days interaction was also significant $[F(5,55)=8.94, p<.01]$, indicating that the rate of mental rotation was faster on Day 2 relative to Day 1 (see Figure 2). Finally, the main effect of replications was statistically significant $[F(17,187)=3.04, p<.01]$, with the pattern of means indicating that identification times actually increased within a given session.

By examining the effect of both days and replications on identification times, it could be argued that the effect of days is attributable to the repetition of the words across days. Within a given day, when words were not repeated, identification times increased. Across days, however, with repetition of words at the same orientation, the identification times decreased. Since it is often argued that mental rotation is not a general skill that can be improved with practice (Cooper \& Podgorny, 1976; Masson, 1986), we

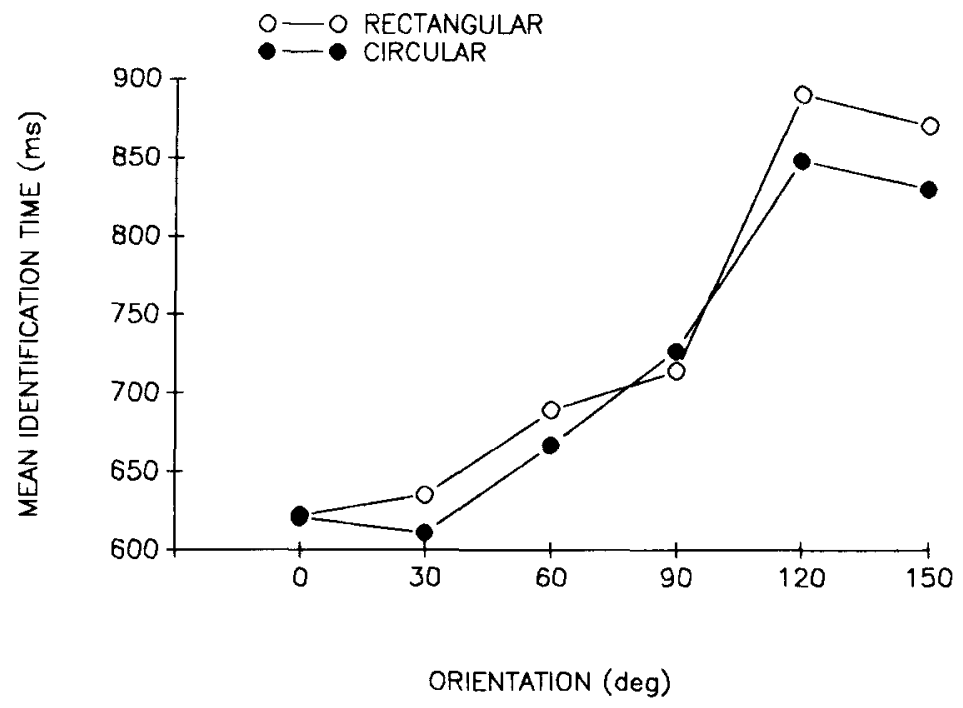

Figure 1. Mean word identification times (in milliseconds) in Experiment 1 as a function of word orientation and viewing frame. 


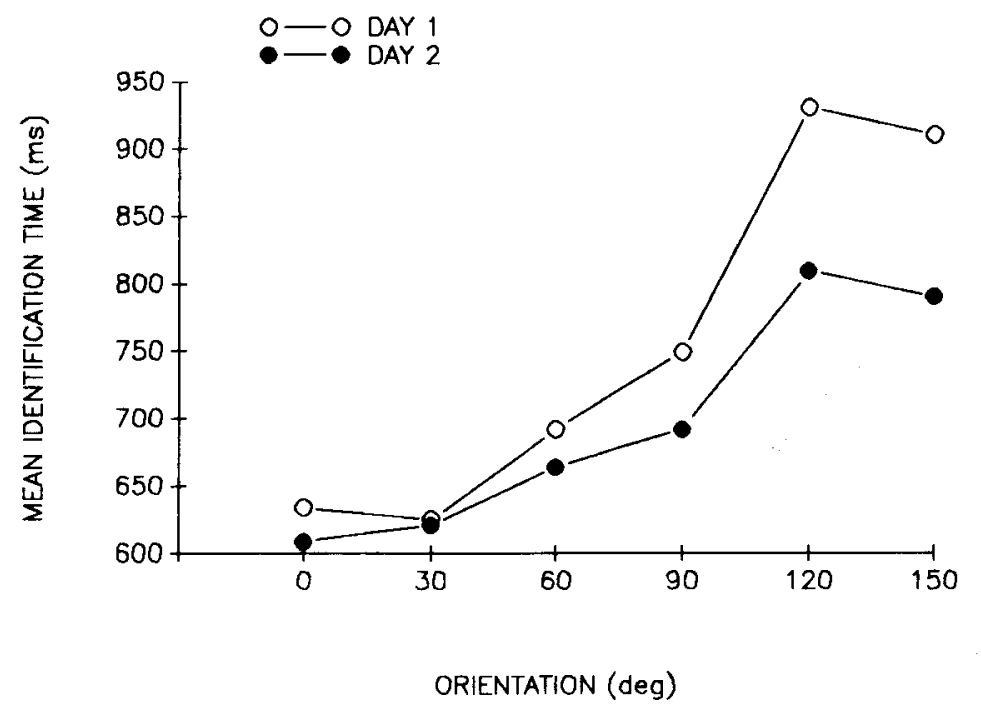

Figure 2. Mean word identification times (in milliseconds) in Experiment 1 as a function of word orientation and session.

would argue that the effect of days is due to "instancebased skill acquisition" (Masson, 1986). According to this argument, the use of the same set of words on both Day 1 and Day 2 improved identification time specific to the word set used, without a general improvement in "mental rotation"' ability.

Finally, as noted above, there was no effect of presenting the misoriented words in either a circular or a rectangular viewing frame $[F(1,10)=1.32]$, nor was there a viewing frame $\times$ orientations interaction $[F(5,50)=1.78$, $p>.10]$, which would have indicated a difference in rates of mental rotation between the two viewing frames. The effect of viewing frame reported by Corballis, Nagourney, et al. (1978) probably reflected individual differences between the two groups of observers, rather than a true effect of frame shape. Thus, providing observers with cues to the local horizontal and vertical axes did not provide for facilitation of mental rotation in this experiment. As noted previously, Corballis, Nagourney, et al. (1978) proposed that a circular frame might slow mental rotation rates by inducing uncertainty about where to mentally rotate misoriented characters. However, our failure to find an influence of frame type might be due to words' having a 'broad range of perceptual uprightness" (see Hock \& Tromley, 1978). That is, observers might imagine the rotation of a misoriented word toward upright until it is close enough to $0^{\circ}$ to allow identification. Hock and Tromley (1978) invoked this "broad tuning" hypothesis to describe the nonlinearity of the "mental rotation" function reported by Cooper and Shepard (1973). The nonlinearity is produced by a "flattening" of the function between $0^{\circ}$ and $60^{\circ}$, which indicates that observers may not need to imagine the rotation of misoriented characters that are close to upright. If there are broadly tuned representations of words around $0^{\circ}$, much of the uncertainty about where to terminate the imagined rotation of the misoriented words presented within a circular frame would be removed.

\section{EXPERIMENT 2}

In Experiment 1, it was hypothesized that if a frame rotation strategy was available to observers, and if frame readjustment was a gradual process of adaptation, then the strategy would emerge with blocked presentation of trials. However, the data indicated that frame rotation was not used by observers. Rather, on each trial, despite the predictability of the upcoming orientation within a block, observers imagined the rotation of the misoriented stimulus to upright. One possible criticism of this conclusion is that observers may have been engaging in frame rotation, but, that, given the relatively long intertrial interval (approximately $3 \mathrm{sec}$ ), observers' reference frames may have defaulted to the upright orientation between trials within a block. For this reason, we shortened the intertrial interval to $20 \mathrm{msec}$ (as did Koriat \& Norman, 1984), to prevent rotation of the reference frame back to the upright, and we combined the shortened intertrial interval with the blocked presentation of trials within a given orientation. If observers have a frame rotation strategy available, these conditions of blocked presentation of trials within an orientation and a shortened intertrial interval should encourage its use.

In addition, in Experiment 2 we used a lexical decision task and orientations ranging from $0^{\circ}$ to $300^{\circ}$ in $60^{\circ}$ steps, in order to facilitate comparison with the data of Koriat and Norman (1984).

Finally, in Experiment 2, half of the observers were exposed to blocks of trials in which all of the letter strings had the same orientation while the other half of the ob- 
servers were exposed to randomized presentation of letter strings and orientations across trials. A comparison of these two conditions of presentation controls for the possibility that observers engage in partial rotation of an internal reference frame on each trial.

\section{Method}

Observers. Twenty-four students from introductory psychology and statistics courses at San Jose State University participated in the experiment in order to earn course credit. None of the observers had participated in Experiment 1.

Stimuli and Apparatus. The observers were asked to make lexical decisions about letter strings presented one at a time on the screen of a Sony CPD-1303 monitor that was $20 \mathrm{~cm}$ high and $28 \mathrm{~cm}$ wide. Stimulus presentation was controlled by an IBM-ATcompatible computer with an 80386 processor. The stimuli were 200 high-frequency words (mean frequency of 215 per million words of text) drawn from Kučera and Francis (1967). One hundred words were four letters long, and the remaining 100 were six letters long. Two hundred nonwords were produced by changing one vowel in each word such that the nonwords were pronounceable and orthographically legal. At the beginning of an observer's session, stimuli were randomly assigned to orientations to control for any effects specific to a given stimulus. No observer saw the same stimulus (word or nonword) more than once. The viewing distance, $78 \mathrm{~cm}$, was maintained by a head- and chinrest. The letter strings were composed of black uppercase letters $\left(1 \mathrm{~cm}\right.$ or $0.7^{\circ}$ high) and were centered on a white background on the monitor. There were six orientations of letter strings $-0^{\circ}$ to $300^{\circ}$, in $60^{\circ}$ steps-relative to the normal, upright orientation.

Procedure. The observers were run individually in a small experimental room under normal fluorescent lighting. They were told to look at the screen and determine whether the letter string presented on each trial was a word or a nonword, as rapidly as possible while maintaining accuracy. They were to signal a response of "word" by pressing a labeled key on the keyboard with the left index finger; a response of "nonword" was to be signaled by pressing a labeled key with the right index finger.

The observers first proceeded through 24 practice trials. A trial consisted of the presentation of the letter string, which remained on the screen until the observer made a response. Twenty milliseconds after the observer's response, the letter string for the next trial appeared.

Following the practice trials, 12 of the 24 observers proceeded through six blocks of 48 trials-one block for each of the six orientations. These observers were told that for all 48 trials within a block, the stimuli would be at the same orientation. As in Experiment 1, these observers were therefore cued to the orientation of the remaining trials in a block by the first trial. Within a block, the 48 trials consisted of the randomized presentation of 24 words and 24 nonwords. The order of presentation of the six blocks of orientations was randomized for each observer in this condition. Error trials were replaced with new stimuli at the end of the block in which they occurred.

For the remaining 12 observers, the practice trials were followed by the randomized presentation of 24 words and 24 nonwords at each of the six orientations. Therefore, these observers were not cued to stimulus orientation at any point during the experiment. Error trials were replaced with new stimuli at the end of the session.

Observers in both conditions participated in 288 trials, responding to 24 words and 24 nonwords at each of the six orientations. The entire procedure took less than $10 \mathrm{~min}$ for each observer.

\section{Results and Discussion}

Lexical decision times were analyzed in a 2 (conditions of presentation of orientations: blocked and random) $\times$
2 (stimulus types: word and nonword) $\times 6$ (orientations) $\times 24$ (replications) $\times 24$ (observers) mixed analysis of variance. Conditions of presentation of orientations was a between-subjects factor.

Figure 3 presents the mean lexical decision times for correct responses for the words (Figure 3A) and the nonwords (Figure 3B) in the blocked and random presentation conditions. As in Experiment 1, the lexical decision times as a function of orientation provide evidence for mental rotation. The main effect of orientation was statistically significant $[F(5,110)=51.08, p<.01]$. In addition, the main effect of stimulus type was significant $[F(1,22)=98.76, p<.01]$. Lexical decision times were an average of $178 \mathrm{msec}$ faster for words than for nonwords. The effects of stimulus orientation and of stimulus type parallel those reported by Koriat and Norman (1984) for lexical decisions about misoriented Hebrew letter strings. The main effect of replications did not reach significance $[F(23,506)<1]$. Since we used a 20 -msec intertrial interval in Experiment 2, the increases in identification time across replications in Experiment 1 may have been due to the difficulty of maintaining vigilance, given the 3-sec intertrial interval.

The error data were analyzed in a 2 (conditions) $\times 2$ (stimulus types) $\times 6$ (orientations) $\times 24$ (observers) mixed analysis of variance. Condition of presentation (blocked and random) was a between-subjects variable. The main effect of orientation was statistically significant $[F(5,110)=10.3, p<.01]$. The highest error rates were at $120^{\circ}$ (1.9 errors per 48 trials) and $240^{\circ}$ ( 2.3 errors per 48 trials), indicating that the lexical decision times do not reflect a speed-accuracy tradeoff.

In order to distinguish the frame and image rotation hypotheses, two statistical results need to be assessed. First, if observers are able to partially readjust an internal reference frame within the blocked condition due to the predictability of orientation, there should be an interaction of conditions of presentation of orientations (blocked and random) and orientation. The slope of the orientation function would be less for the blocked presentation condition than for the random presentation condition. In the mixed analysis of variance described above, the interaction of condition and orientation was statistically significant $[F(5,110)=3.07, p<.01]$. However, the slope difference was in a direction opposite that predicted by a partial frame rotation hypothesis; that is, the slope was greater for the blocked condition than for the random condition. Second, as in Experiment 1, a frame rotation strategy would be indicated by an orientation $\times$ replications interaction for the blocked presentation condition. The slope of the orientation function would decrease across replications if observers gradually adjusted an internal reference frame within a block of 48 trials in which all stimuli had the same orientation. In a 2 (stimulus types) $\times 6$ (orientations) $\times 24$ (replications) $\times 12$ (observers) within-subjects analysis of variance of the lexical decision times in the blocked presentation condition, the orientation $\times$ replications interaction did not reach statistical significance $[F(115,1265)=1.08]$. 
Thus, the present experiment combined a shortened intertrial interval (to prevent the internal reference frame from defaulting to the normal, upright orientation; see Koriat \& Norman, 1984) with blocks of 48 trials (to allow for a gradual readjustment of the internal reference frame). Under these conditions, there was again no evidence of a frame rotation strategy, despite the claim of Koriat and Norman (1984) and Robertson et al. (1987) that both image and frame rotation strategies are available to observers. Rather, the data indicated that observers continue to imagine the rotation of a misoriented stimu- lus to upright prior to deciding its lexical status. In the next experiment, we increased the number of trials within a block from 48 to 96 , to test the possibility that the readjustment of an internal reference frame takes more than 48 trials.

\section{EXPERIMENT 3}

In both Experiments 1 and 2, the data indicated that observers engaged in image rotation in order to identify (Experiment 1) or decide the lexical status of (Experiment 2)

(A)

WORDS - 24 TRIALS

O-O BLOCKED

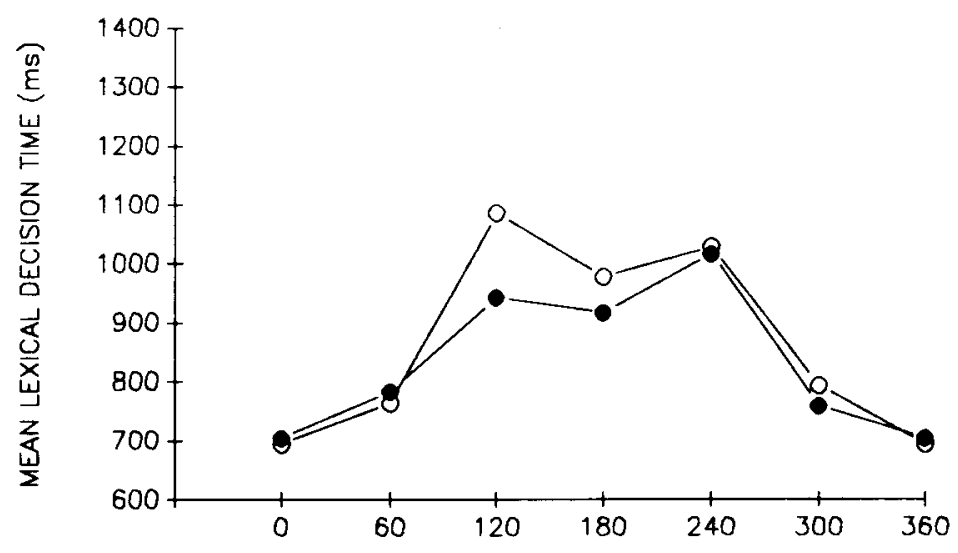

(B)

NONWOROS - 24 TRIALS

$O-O$ BLOCKED

RANDOM

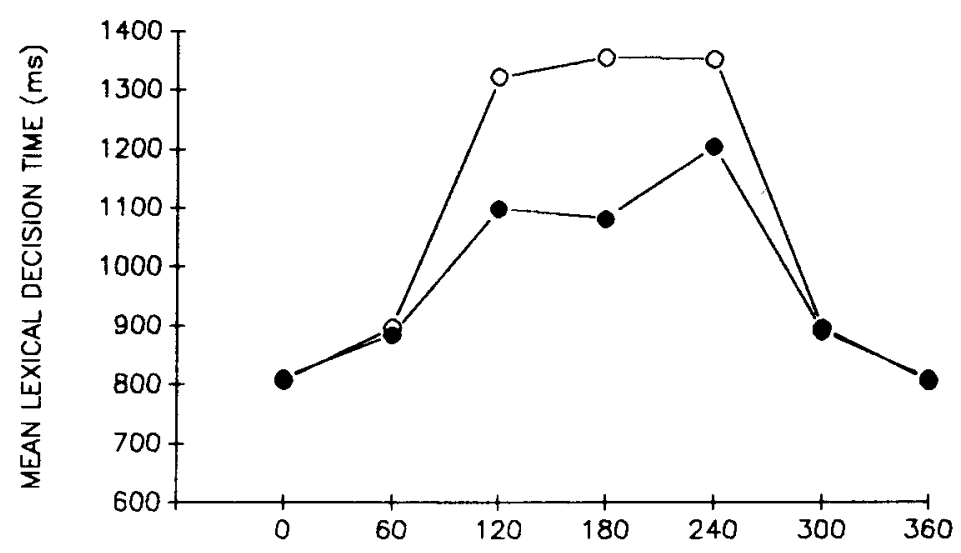

ORIENTATION (deg)

Figure 3. Mean lexical decision times (in milliseconds) in Experiment 2 as a function of (A) word orientation and presentation condition and (B) nonword orientation and presentation condition. 
misoriented letter strings. These data were obtained under conditions that should have encouraged the use of a frame rotation strategy if it was available to the observer. In this final experiment, the number of consecutive trials of the same orientation was increased to 96 , in order to assess whether or not the results of the previous experiments represent a Type II error; that is, the process of frame readjustment might be even more gradual than we had allowed for in the previous experiments.

\section{Method}

Observers. Twenty-four students from an introductory psychology course at San Jose State University participated in the experiment in order to earn course credit. None of the observers had participated in Experiment 1 or 2.

Stimuli and Apparatus. The stimuli and apparatus used in the present experiment were identical to those in Experiment 2, with the following exception: Instead of the six orientations used in Experiment $2\left(0^{\circ}\right.$ to $360^{\circ}$, in $60^{\circ}$ steps $)$, only three orientations $\left(0^{\circ}\right.$, $120^{\circ}$, and $240^{\circ}$ ) were used in Experiment 3.

Procedure. The procedure for Experiment 3 was identical to that of Experiment 2, with the exception of the number of orientations sampled and the number of trials within a given orientation.

Following the 24 practice trials, 12 of the 24 observers proceeded through three blocks of 96 trials-one block for each of the three orientations. These observers were told that for all 96 trials within a block, the stimuli would be at the same orientation. Within a block, the 96 trials consisted of the randomized presentation of 48 words and 48 nonwords. The order of presentation of the three blocks of orientations was randomized for each observer in this condition. Error trials were replaced with new stimuli at the end of the block in which they occurred.

For the remaining 12 observers, the practice trials were followed by the randomized presentation of 48 words and 48 nonwords at each of the three orientations. Error trials were replaced with new stimuli at the end of the session.

Observers in both conditions participated in 288 trials, responding to 48 words and 48 nonwords at each of the three orientations. The entire procedure took less than $10 \mathrm{~min}$ for each observer. Again, no observer saw the same stimulus (word or nonword) more than once.

\section{Results and Discussion}

Lexical decision times were analyzed in a 2 (conditions of presentation of orientations: blocked and random) $\times$ 2 (stimulus types: word and nonword) $\times 3$ (orientations) $\times 48$ (replications) $\times 24$ (observers) mixed analysis of variance. Condition of presentation of orientations was a between-subjects factor.

Figure 4 presents the mean lexical decision times for correct responses for the words (Figure 4A) and the nonwords (Figure $4 \mathrm{~b}$ ) in the blocked and random presentation conditions. The lexical decision times as a function of orientation provide evidence for mental rotation. The main effect of orientation was statistically significant $[F(2,44)=52.05, p<.01]$. As in Experiment 2, the main effect of stimulus type was significant $[F(1,22)=$ $178.44, p<.01]$. Lexical decision times were an average of $219 \mathrm{msec}$ faster for words than for nonwords. As in Experiment 2, the main effect of replications did not reach significance $[F(47,1034)<1]$, nor did replications interact with any other factor.

The error data were analyzed in a 2 (conditions) $\times 2$ (stimulus types) $\times 3$ (orientations) $\times 24$ (observers) mixed analysis of variance. Condition of presentation (blocked and random) was a between-subjects variable. The main effect of orientation was statistically significant $[F(2,44)=7.1, p<.01]$. The highest error rate was at $240^{\circ}$ (5.7 errors per 96 trials), indicating that the lexical decision times do not reflect a speed-accuracy tradeoff.

As in Experiment 2, two statistical results need to be assessed to distinguish between image and frame rotation hypotheses. First, in order to test the possibility that observers partially readjust an internal reference frame within the blocked condition due to the predictability of orientation, we tested for an interaction of conditions of presentation of orientations (blocked and random) and orientation. In the mixed analysis of variance described above, this interaction of condition and orientation did not reach statistical significance $[F(2,44)<1]$. Second, if observers gradually readjust an internal frame of reference within a block of 96 trials at the same orientation, the slope of the orientation function would decrease across replications. In a 2 (stimulus types) $\times 3$ (orientations) $\times 48$ (replications) $\times 12$ (observers) within-subjects analysis of variance of the lexical decision times for the blocked presentation condition, the orientation $\times$ replications interaction did not reach statistical significance $[F(94,1034)<1]$. Once again, Experiment 3 indicates that observers imagine the rotation of a misoriented letter string to upright for further processing rather than fully or partially rotating an internal frame of reference to the misoriented stimulus.

\section{GENERAL DISCUSSION}

There were three principal findings in the present studies. First, a function implying "mental rotation" was observed in all three experiments, using the stimulustask combinations of identification of (Experiment 1) or lexical decisions about (Experiments 2 and 3) highfrequency English words. Second, this function persisted within a block of trials in which the orientation of all stimuli was the same, a finding that supports an image rotation, rather than a frame rotation, hypothesis. Finally, identification times were faster on the 2nd day of Experiment 1 . Since the same set of words was used on both days, this result could indicate that the improvement in the identification of misoriented words was specific to the word set used. These results will be discussed in turn.

\section{The Mental Rotation of Words}

The data of the present experiments extend the work of Koriat and Norman (1984) involving lexical decisions about misoriented Hebrew letter strings to both the identification of and lexical decisions about misoriented En- 
(A)

WORDS - 48 TRIALS
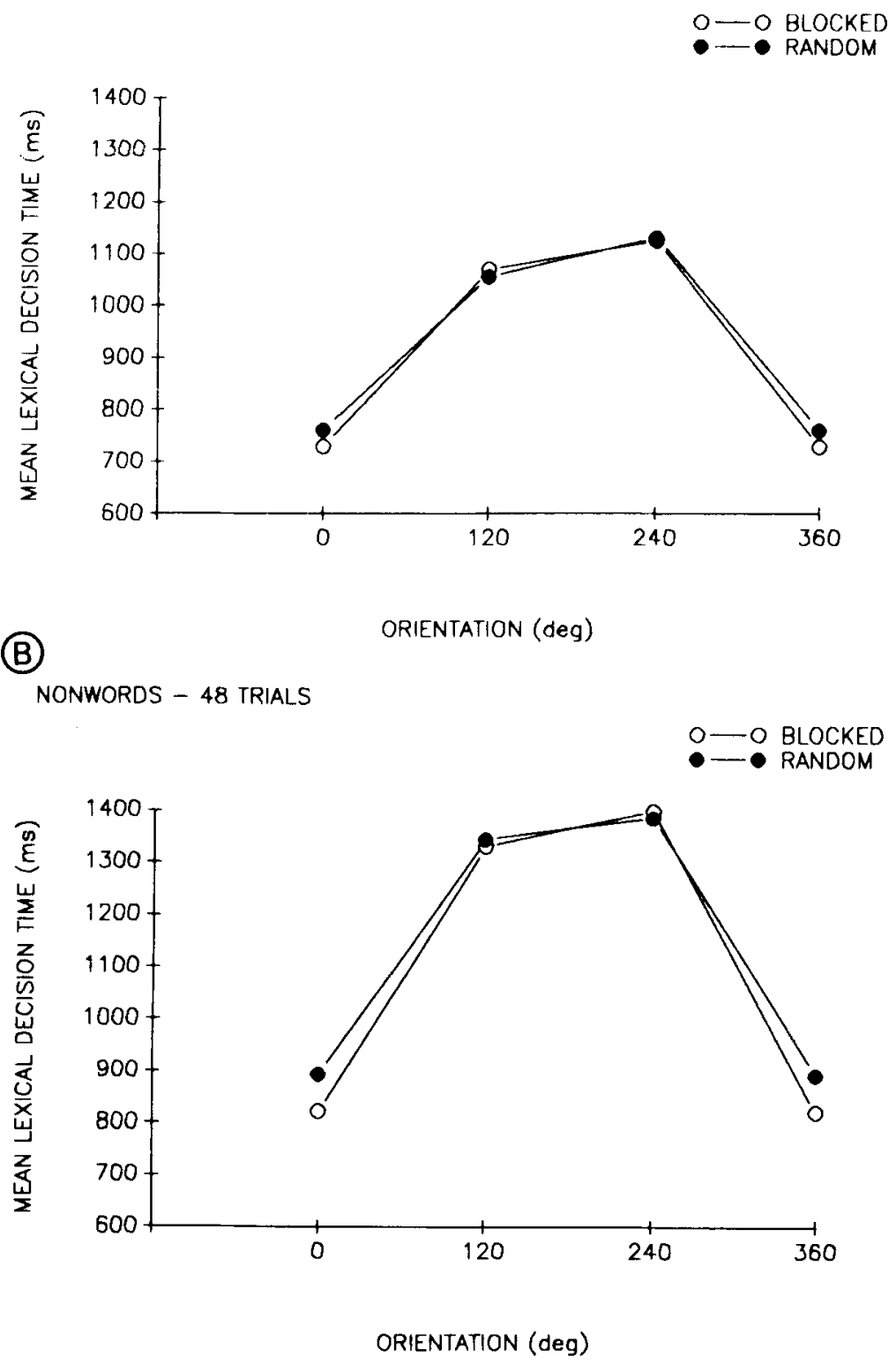

Figure 4. Mean lexical decision times (in milliseconds) in Experiment 3 as a function of $(A)$ word orientation and presentation condition and $(B)$ nonword orientation and presentation condition.

glish words. In particular, Experiment 2 was done with the same orientations and task as those of Koriat and Norman, and the results of the orientation and stimulus type manipulations were nearly identical to those of Koriat and Norman.

In addition, the shape of the function in both experiments satisfies the criterion for mental rotation suggested by Corballis and Cullen (1986). In fact, the estimated rotation rates were close to those of Cooper and Shepard (1973). Finally, the time to identify misoriented words did not decrease within any session of the three experi- ments, a result that is consistent with Cooper and Podgorny's (1976) claim that the rate of imagined rotation of stimuli does not increase with practice.

\section{The Image Rotation Hypothesis}

All three of the present experiments were designed to test whether the function relating response time to stimulus orientation reflects an imagined rotation of a stimulus to upright (the "image rotation" hypothesis) or of a perceptual reference frame to the orientation of the stimulus (the "frame rotation" hypothesis). In previous studies that 
had provided minimal support for the frame rotation hypothesis (Koriat \& Norman, 1984; Robertson et al., 1987), these hypotheses were investigated by analyzing response times to misoriented stimuli as a function of the angular departure of the stimulus from upright (ADU) or the angular departure from the preceding stimulus orientation (ADP). The finding of a minimal effect of ADP was interpreted to indicate that observers have both image and frame rotation strategies available, and that they use the strategy which is more expedient (Koriat \& Norman, 1984; Robertson et al., 1987).

The important difference between the present experiments and the previous studies of image and frame rotation was that there was no trial-to-trial uncertainty in the present experiments. The use of blocks of trials in which all stimuli were of the same orientation and the use of short intertrial intervals (in Experiments 2 and 3) should have allowed for the possibility of a gradual shift in the orientation of a subjective reference frame (adaptation) if the frame rotation strategy was available.

In order to understand the failure to find an effect of ADP (and to understand the effect of ADP itself) on response time in the present experiments, it is useful to consider a recent reexamination of the Koriat and Norman (1984) data that showed an effect of ADP (Experiment 1). Koriat and Norman (1988) had reported that this effect of ADP on the time to make a decision about the version (normal or mirror image) of a misoriented Hebrew letter was almost entirely attributable to situations in which the preceding trial stimulus was the "same letter in the same format"-for example, when Trials 1 and 2 both contained a normal version of the same Hebrew letter. Koriat and Norman (1988) have also pointed out that the data indicating an effect of ADP in the Robertson et al. (1987) studies were also restricted to same-letter-sameformat trials and to a lesser extent to "different letter same format" trials in which the letters were quite similar (e.g., F and R; see Koriat \& Norman, 1988, p. 110). Thus, the processing that occurred on the previous trial led to savings during processing of the same (or a similar) stimulus on the present trial. Rather than a readjustment of an internal reference frame, the ADP effect might simply reflect a "backward alignment"' (Koriat \& Norman, 1988, 1989a) of the presently viewed stimulus into congruence with a short-lived trace of the same stimulus viewed on the previous trial. Alternatively, effects of ADP might also indicate that when observers are exposed to a misoriented version of a stimulus, they form an additional, longer lasting, stored representation of the stimulus at the novel orientation. When subsequently presented with that same stimulus at an orientation close to the novel orientation, observers can imagine the rotation of the stimulus into congruence with the newly formed representation for further processing (the "mental transformation" and "multiple views" hypotheses; see Tarr \& Pinker, 1989).

Neither of the above descriptions invokes a readjustment of an internal reference frame to describe either ADP effects or the "mental rotation" function. Rather, the data of Koriat and Norman $(1984,1988)$, Robertson et al. (1987), and the present experiments lend overwhelming support to the conclusion of Shepard and his colleagues (see Shepard \& Cooper, 1982) that when an observer is faced with a misoriented stimulus, the observer will engage in a process of imagining the rotation of the stimulus to upright for further processing.

\section{The "Days" Effect and Stimulus Repetition}

In Experiment 1, the slope of the function relating identification time to word orientation decreased from the 1st to the 2nd day. It is important that the same set of words was used at the same orientation each day. In order to understand this slope change, it must be noted that the function relating stimulus orientation to reaction time often depends on the choice of stimuli and the task of the observer (see Koriat \& Norman, 1989b). For example, Corballis, Zbrodoff, Shetzer, and Butler (1978) combined the six-item alphanumeric character set used by Cooper and Shepard (1973) as stimuli with an identification task. There was some initial indication of increased identification time with the individual stimuli presented singly as the angular departure from upright increased to $180^{\circ}$ (Block 1 of their Experiment 1), but the function quickly "flattened" out (Corballis, Zbrodoff, et al. 1978, Figure 1, p. 100); that is, identification time was independent of stimulus orientation. In addition, White (1980) reported that the orientation of alphanumeric characters did not affect the time to name the characters, and Koriat and Norman (1989b) reported that the orientation of Hebrew characters did not affect identification time. Eley (1982) pointed out that these results indicate a featureextraction process that is independent of orientation. This feature-extraction process would be facilitated by the small stimulus set (six items) used by Corballis, Zbrodoff, et al. (1978) and by the fact that the alphanumeric stimuli were highly familiar, overlearned stimuli. Thus, according to the criteria of Corballis and Cullen (1986) and Cooper and Podgorny (1976) presented above, it seems that mental rotation may not be necessary in alphanumeric identification.

One regularity that emerges from the studies of Corballis, Zbrodoff, et al. (1978), Koriat and Norman (1989b), and White (1980) is that repetition of familiar, but misoriented, stimuli in an identification task reduces the slope of the function that relates identification time to stimulus orientation; this either increases the estimated rate of, or eliminates the need for, mental rotation. Consistent with this observation, Jolicoeur (1985; see also Jolicoeur \& Milliken, 1989) reported that the time to name misoriented natural objects (e.g., an outline drawing of a lamp) initially depended on object orientation, but that this effect of orientation quickly diminished with practice. It is important that Jolicoeur reported that the savings in naming time were specific to the object set used; the reduction of the effect of object orientation on naming time did not transfer to a different set of objects. In addition, Masson (1986) reported a reduction in reading 
time for misoriented text, which did not transfer to new text or to the same text in a different case (e.g., upper vs. lower case). Masson argued that these results indicated that improvement in reaction times within a mental rotation paradigm is due to learning based on specific instances, a process that would be facilitated by repeated exposure to the same stimulus set. In fact, Masson states that "word identification skill develops through memory for analyses of specific instances to which a subject is exposed" (p. 479). In the absence of repeated exposure to specific instances, continuous use of mental rotation is expected in a word identification task such as that used in the present studies.

Many of the results of the present experiments are consistent with the Jolicoeur (1985; Jolicoeur \& Milliken, 1989) and Masson (1986) findings. In the first session of Experiment 1, observers identified misoriented highfrequency words. There was a substantial amount of practice (18 replications) at each orientation. It is important that the practice was not instance-based, in that observers never identified a given stimulus more than once. If mental rotation is a general skill (in Masson's terms) that is unaffected by practice (Cooper \& Podgorny, 1976), any effect of misorientation would be expected to persist despite practice. This general skill hypothesis is supported by the data of the first session of Experiment 1 and those of Experiments 2 and 3. A function indicating mental rotation was observed, and it did not "flatten"; identification times did not decrease across 18 replications within a session in Experiment 1, nor did lexical decision times decrease across 48 replications in Experiment 2 and across 96 replications in Experiment 3. However, in the second session of Experiment 1, there was a significant reduction in the slope of the mental rotation function (the days $\times$ orientation interaction; see Figure 2). This effect may have been due to the repetition of the words across days. The practice from Day 1 to Day 2 was instance-based in that the same words were presented at the same orientations in the second session.

We have tested this instance-based-skill acquisition hypothesis as it relates to mental rotation, using a transfer paradigm in which observers participated in two sessions (Jordan \& Huntsman, 1990). In the first session, observers made lexical decisions about misoriented letter strings. The second session contained the same set of letter strings at the same orientation used in the first session and the same set of letter strings at a different orientation (rotated $180^{\circ}$ ), as well as a novel set of letter strings. The function relating lexical decision times to orientation flattened (i.e., there was no mental rotation function) only for the condition in which the same words were repeated at the same orientation. For the other two conditions, the characteristic mental rotation function was observed.

\section{Summary}

The present experiments indicate that when observers are confronted with misoriented English letter strings, they imagine the rotation of the stimulus to upright for further processing (the image rotation hypothesis). However, when a word has been previously seen at a particular misorientation, observers do not seem to engage in mental rotation. Eley (1982) contended that the lack of mental rotation observed with alphanumerics reflects orientationinvariant feature extraction. For example, with practice, it might be easy to detect the orientation-invariant features that distinguish a $\mathbf{G}$ from an $\mathrm{R}$. With more complex stimuli, such as words (Jordan \& Huntsman, 1990; Masson, 1986; the present studies) or drawings of natural objects (Jolicoeur, 1985; Jolicoeur \& Milliken, 1989), it is not clear which orientation-invariant features distinguish one word (for example) from another and allow for orientation-invariant reaction times. It might be, as Tarr and Pinker (1989) proposed, that when a misoriented stimulus is first presented to an observer, the observer forms an additional stored representation of the stimulus at that orientation. On subsequent presentation of the stimulus at the same orientation, the observer can match the misoriented stimulus to this newly formed representation, thus eliminating the need for mental rotation.

\section{REFERENCES}

CoOper, L. A., \& Podgorny, P. (1976). Mental transformations and visual comparison processes: Effects of complexity and similarity. Journal of Experimental Psychology: Human Perception \& Performance, 2, 503-514.

CoOper, L. A., Shepard, R. N. (1973). Chronometric studies of the rotation of mental images. In W. G. Chase (Ed.), Visual information processing (pp. 75-175). New York: Academic Press.

Corballis, M. C., \& Cullen, S. (1986). Decisions about the axes of disoriented shapes. Memory \& Cognition, 14, 27-38.

Corballis, M. C., Nagourney, B. A., Shetzer, L. I., \& Stephanatos, G. (1978). Mental rotation under head tilt: Factors influencing the location of the subjective reference frame. Perception \& Psychophysics, 24, 263-273.

Corballis, M. C., Zbrodoff, J., \& Roldan, C. E. (1976). What's up in mental rotation? Perception \& Psychophysics, 19, 525-530.

Corballis, M. C., Zbrodoff, N. J., Shetzer, L. I., Butler, P. B. (1978). Decisions about identity and orientation of rotated letters and digits. Memory \& Cognition, 6, 98-107.

ELEY, M. G. (1982). Identifying rotated letter-like symbols. Memory \& Cognition, 10, 25-32.

Hock, H. S., \& TROMLEY, C. L. (1978). Mental rotation and perceptual uprightness. Perception \& Psychophysics, 24, 529-533.

JolicoeUR, P. (1985). The time to name disoriented natural objects. Memory \& Cognition, 13, 289-303.

JOLICOEUR, P., \& MILLIKEN, B. (1989). Identification of disoriented objects: Effects of context of prior presentation. Journal of Experimental Psychology: Learning, Memory, \& Cognition, 15, 200-210.

JORDAN, K. (1987, November). Identification times for misoriented words. Paper presented at the annual meeting of the Psychonomic Society, Seattle.

JoRdan, K., \& Huntsman, L. (1990). Orientation-specific transfer in a lexical decision task. Manuscript in preparation.

Koriat, A., Norman, J. (1984). What is rotated in mental rotation? Journal of Experimental Psychology: Learning, Memory, \& Cognition, 10, 421-434.

KorIat, A., \& Norman, J. (1988). Frames and images: Sequential effects in mental rotation. Journal of Experimental Psychology: Leaming, Memory, \& Cognition, 14, 93-111.

Koriat, A., \& Norman, J. (1989a). Establishing global and local cor- 
respondence between successive stimuli: The holistic nature of backward alignment. Joumal of Experimental Psychology: Learning, Memory, \& Cognition, 15, 480-494.

Koriat, A., \& Norman, J. (1989b). Why is word recognition impaired by disorientation while the identification of single letters is not? Journal of Experimental Psychology: Human Perception \& Performance, 15, 153-163.

KuČERA, H., \& Francis, W. N. (1967). Computational analysis of present-day American English. Providence, RI: Brown University Press.

Masson, M. E. J. (1986). Identification of typographically transformed words: Instance-based skill acquisition. Journal of Experimental Psychology: Learning, Memory, \& Cognition, 12, 479-488.

Robertson, L. C., Palmer, S. E., \& Gomez, L. M. (1987). Refer- ence frames in mental rotation. Journal of Experimental Psychology: Learning, Memory, \& Cognition, 13, 368-379.

Shepard, R. N., CoOPER, L. A. (1982). Mental images and their transformation. Cambridge, MA: MIT Press.

TARR, M. J., \& PINKer, S. (1989). Mental rotation and orientationdependence in shape recognition. Cognitive Psychology, 21, 233-282.

WHITE, M. J. (1980). Naming and categorization of tilted alphanumeric characters do not require mental rotation. Bulletin of the Psychonomic Society, 15, 153-156.

ZeChMEISTER, E. B., NYBERG, S. E. (1982). Human memory. Monterey, CA: Brooks/Cole.

(Manuscript received November 4, 1988; revision accepted for publication May 17, 1990.)

\title{
Announcement
}

\author{
Cognitive Aspects of Human Audition \\ A Tutorial Workshop \\ Centre National d'Art et de Culture "Georges Pompidou," Paris \\ February 21-22, 1991
}

In recognition of the progress that is being made in research in nonverbal auditory cognition, this workshop was organized by the Hearing Group of the French Acoustical Society and the Institut de Recherche et Coordination Acoustique/Musique (IRCAM) to present the state of the art in the cognitive aspects of sensory information processing. It will be oriented toward students in the cognitive sciences and scientists specializing in fields other than auditory psychology.

There will be four one-hour presentations per day, each followed by a half-hour discussion period:

1. Introduction to auditory cognition-Stephen McAdams (Laboratory of Experimental Psychology, René Descartes University, Paris)

2. Auditory scene analysis: Listening in complex environments-Albert S. Bregman (McGill University, Montreal)

3. Perception of global properties of sound sequences-Richard M. Warren (University of Wisconsin, Milwaukee)

4. Attending to auditory events-Mari Riess Jones (Ohio State University, Columbus)

5. Auditory memory-Robert G. Crowder (Yale University, New Haven)

6. Contributions of music psychology to research on human auditory perception and cognition-Emmanuel Bigand (University of Paris X, Nanterre, France)

7. Recognition of nonlinguistic auditory objects: Contributions from neuropsychology-Isabelle Peretz (University of Montreal)

8. Listening strategies in infancy: The roots of language and musical development-Sandra E. Trehub (Center for Research in Human Development, University of Toronto)

Presentations 1, 6, and 7 will be in French; presentations 2, 3, 4, 5, and 8 will be in English.

For further information and registration forms, write to: Groupe Audition de la S.F.A., Laboratoire de Psychologie Expérimentale, 28, rue Serpente, F-75006 Paris, France. 